\section{Erfolgreicher Widerspruch - KV muss auch Anwaltskosten tragen}

Im Zweifelsfall ist rechtlicher Beistand immer empfehlenswert. Wenn ein Arzt mit seiner KV um viel Geld streitet, gibt es kaum einen Grund auf den Rechtsanwalt zu verzichten.

S treitet ein Arzt mit seiner KV um viel Geld, dann darf er sich in der Regel auch rechtlichen Beistand holen. Soweit die KV nachgibt, muss sie dann auch im Widerspruchsverfahren den Anwalt bezahlen, urteilte kürzlich der Vertragsarztsenat des Bundessozialgerichts (BSG) in Kassel (Aktenzeichen: B 6 KA 43/11 R).

\section{Rechtsbeistand bei großer wirtschaftlicher Tragweite}

Im konkreten Fall hatte die KV BadenWürttemberg die Abrechnungen eines Radiologen für 2003 und 2004 sachlichrechnerisch richtiggestellt und danach fast $155.000 €$ zurückverlangt. Für das Widerspruchsverfahren ließ sich der Arzt von einem Anwalt beraten. Der Widerspruch war auch in vollem Umfang erfolgreich. Allerdings weigerte sich die KV, auch den Rechtsanwalt zu bezahlen. Das muss sie aber, mahnte das BSG. Ob die Hinzuziehung eines Anwalts notwendig war und daher von der unterlegenen KV zu bezahlen ist, sei immer aus der Sicht des Arztes zu entscheiden. Das sei in der Regel zu bejahen, wenn wie hier der Streit „von nicht unerheblicher wirtschaftlicher Tragweite ist". Gleiches gelte, wenn es um "Auslegungsfragen zu den Leistungslegenden der Gebührenordnungen" geht.

Verzichtbar sei ein Rechtsanwalt lediglich dann, wenn der Arzt in der Vergangenheit bereits Erfahrungen in der strittigen Frage gemacht hat oder wenn es um einen offensichtlichen Fehler der KV geht, auf den der Arzt lediglich hinweisen muss. Dagegen spiele es keine Rolle, ob der Anwalt im Widerspruchsverfahren eine eigene Stellungnahme abgegeben hat, betonten die Kasseler Richter.

Martin Wortmann

\section{Abrechnungstipp!}

\section{Häufiger Beschwerdeanlass - die Nr. 2 bei Terminvereinbarungen}

- Immer wieder beanstanden Patienten, dass bereits bei alleiniger Terminvereinbarung eine Gebühr, in der Regel nach Nr. 2 GOÄ, abgerechnet wird. Wenn es sich nachweislich um eine reine Terminvereinbarung handelt, ist die Beanstandung korrekt, denn die Nr. 2 beinhaltet die „Ausstellung von Wiederholungsrezepten und/oder Überweisungen und/oder Übermittlung von Befunden oder ärztlichen Anordnungen - auch mittels Fernsprecher - durch die Arzthelferin und/oder Messung von Körperzuständen (z.B. Blutdruck, Temperatur) ohne Beratung, bei einer Inanspruchnahme des Arztes."

Die alleinige Terminvereinbarung ist nicht Leistungsbestandteil der Nr. 2. Häufig ist es aber vor allem bei akuten Erkrankungen der Fall, dass aufgrund vorgegebener Behandlungsanweisungen in der Praxis die Mitarbeiterin dem/der Patienten/in Verhaltensmaßregeln gibt, wie im aktuellen Fall zu reagieren ist, bis ein Termin und damit ein Arzt-Patienten-Kontakt zustande kommt. Dies kann durchaus auch noch am selben Tage geschehen. Da der Patient sich allerdings häufig nur an die Terminvereinbarung erinnert, ist es zwingend notwendig, die Vermittlung dieser Verhaltensmaßregeln durch die Praxismitarbeiterin in der Patientendatei zu dokumentieren. Nur so kann oft schon beim Gespräch mit dem sich beschwerenden Patienten die Angelegenheit geklärt werden.

Dr. med. Heiner Pasch

\section{Ärztebewertung im Netz - Gericht setzt Grenzen}

Grundsätzlich müssen Ärzte Kritik und Bewertungen im Internet hinnehmen. Aber: Wenn ein Patient dort falsche Angaben über eine Behandlung abgibt, können Ärzte dagegen vorgehen (Aktenzeichen 11 O 2608/12). Im Fall eines Zahnarztes haben Richter jetzt einem Portalbetreiber deutliche Auflagen gemacht. Ein Patient hatte sich über eine Implantatbehandlung beschwert. Der Zahnarzt konterte, in dem angegebenen Zeitraum habe er gar keine Implantatbehandlung durchgeführt und forderte die Löschung der Vorwürfe im Netz. Das Landgericht Nürnberg-Fürth gab ihm Recht.

\section{Martin Wortmann}

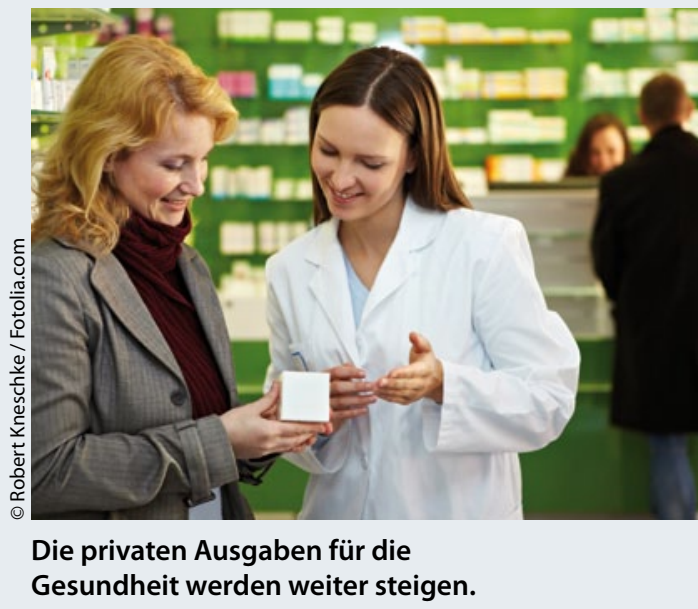

\section{Medical Wellness und Prävention sind Zukunftstrends}

Nach einer Studie der Berenberg Bank und des Hamburgischen Weltwirtschaftsinstituts (HWWI) steigt der Trend zu Medical Wellness und Prävention. Privat finanzierte Gesundheitsausgaben werden in Zukunft weiter wachsen. Die Weltbevölkerung wächst, die westlichen Gesellschaften altern, die Einkommen steigen und die Gesundheitsausgaben nehmen überproportional zum Einkommen zu, prognostizieren die Wirtschaftsforscher. Mit privaten Ausgaben sorgen schon jetzt viele Menschen dafür, dass die Wachstumsraten im Gesundheitsmarkt deutlich über dem des durchschnittlichen Wirtschaftswachstums liegen.

Dirk Schnack 